\title{
Neural Recommender System for Activity Coefficient Prediction and UNIFAC Model Extension of Ionic Liquid-Solute Systems
}

\author{
Guzhong Chen ${ }^{1}$, Zhen Song ${ }^{2}$, Zhiwen Qi ${ }^{1}$, and Kai Sundmacher ${ }^{3}$ \\ ${ }^{1}$ East China University of Science and Technology \\ ${ }^{2}$ Max-Planck-Institut fur Dynamik komplexer technischer Systeme \\ ${ }^{3}$ Max Planck Institute for Dynamics of Complex Technical Systems
}

July 21,2020

\begin{abstract}
For the ionic liquid (IL)-solute systems of broad interest, a deep neural network based recommender system (RS) for predicting the infinite dilution activity coefficient $(\gamma[?])$ is proposed and applied for a large extension of the UNIFAC model. In the RS, neural network entity embeddings are employed for mapping each IL and solute and neural collaborative filtering is utilized to handle the nonlinearities of IL-solute interactions. A comprehensive experimental $\gamma[$ ?] database covering 215 ILs and 112 solutes (totally 41,553 data points) is established for training the RS, where the cross-validation and test are performed based on a rigorous dataset split by IL-solute combinations. The obtained RS shows superior performance than the state-of-the-art $\curlyvee[?]$ models and is thus taken to complete the solute-in-IL $\curlyvee[?]$ matrix. Based on the completed $\gamma$ [?] database, a large extension of the UNIFAC-IL model is finally presented, filling all the parameters between involved groups.
\end{abstract}

\section{Hosted file}

AICHE-Manuscript-20200715.docx available at https://authorea.com/users/344984/articles/ 471361-neural-recommender-system-for-activity-coefficient-prediction-and-unifac-modelextension-of-ionic-liquid-solute-systems 